\title{
The influence of prostatic Cutibacterium acnes infection on serum levels of IL6 and CXCL8 in prostate cancer patients
}

\author{
Henrik Ugge ${ }^{1 *} \mathbb{D}$, Jessica Carlsson ${ }^{1}$, Bo Söderquist ${ }^{2}$, Katja Fall ${ }^{3,4}$, Ove Andén ${ }^{1}$ and Sabina Davidsson ${ }^{1}$
}

\begin{abstract}
Background: Chronic prostatic inflammation, caused by Cutibacterium acnes (C. acnes), has been proposed to influence the risk of prostate cancer development. In vitro studies have demonstrated the capacity of $C$. acnes to induce secretion of Interleukin 6 (IL6) and C-X-C motif chemokine ligand 8 (CXCL8) by prostate epithelial cells. Both these inflammatory mediators have been implicated in prostate cancer pathophysiology. In this cohort study, we aimed to investigate the influence of prostatic C. acnes on serum levels of IL6 and CXCL8.

Methods: We recruited 99 prostate cancer patients who underwent radical prostatectomy at Örebro University Hospital. The cultivation of pre-operatively obtained prostate biopsies identified C. acnes in 60 of the 99 patients. Levels of IL6 and CXCL8 in pre-operative serum samples were analyzed using ELISA, and concentrations were compared between prostate cancer patients with and without prostatic C. acnes infection using standard statistical methods.
\end{abstract}

Results: No statistical differences were observed in serum levels of IL6 and CXCL8 between subjects with and without prostatic $C$. acnes infection.

Conclusions: Our results indicate that prostatic $C$. acnes infection may give rise to low-grade inflammation with little effect on systemic levels of IL6 and CXCL8.

Keywords: Prostate cancer, Inflammation, Cutibacterium acnes, IL6, CXCL8, Cytokines

\section{Introduction}

Inflammation is recognized as a critical component of tumor progression and may be involved in the development of several types of cancer [1]. It has further been hypothesized to influence both prostate cancer development and progression [2]. Both acute and chronic inflammation are frequently observed in prostate cancer specimens obtained from biopsy sampling, transurethral prostate resection and prostatectomies [3-5].

The inflammatory microenvironment is believed to contribute to the development of prostate cancer via a number of mechanisms, including production of reactive molecule species with known capacity to cause DNA damage [1]. In addition, increased levels of specific

\footnotetext{
* Correspondence: henrik.ugge@regionorebrolan.se

${ }^{1}$ Department of Urology, Faculty of Medicine and Health, Örebro University, 70185 Örebro, Sweden

Full list of author information is available at the end of the article
}

cytokines and chemokines in a persistently inflamed tissue have been suggested to contribute to prostate cancer initiation, promotion, and progression $[2,6]$. Two of the most frequently studied cytokines and chemokines in relation to prostate cancer development and pathophysiology are Interleukin-6 (IL6) and C-X-C motif chemokine ligand 8 (CXCL8). Several lines of evidence have suggested associations between IL6 and CXCL8, and prostate cancer aggressiveness, progression, and poor clinical outcome [2, 6-11].

The commonly observed prostatic inflammation may be caused by different etiological factors, including infectious agents such as bacteria. Cutibacterium acnes (C. acnes), formerly Propionibacterium acnes, is an anaerobic Gram-positive rod-shaped bacterium, generally regarded as commensal of the skin, but also commonly associated with the skin condition acne vulgaris [12]. $C$. acnes has further been identified as the etiological agent 
in a number of low-grade infections; predominantly implant-associated infections, related to for example prosthetic joint devices, prosthetic heart valves and neurosurgical shunts [13].

An impact of C. acnes in prostate pathologies has been suggested, since several independent studies have observed it to be the most prevalent microorganism in prostate tissue [14-16]. Cohen et al. detected C. acnes in $35 \%$ of cultures from prostatectomy samples, and observed a significantly higher degree of inflammation in samples positive for $C$. acnes compared to negative samples [17]. These findings raised the question whether $C$. acnes could be involved in inflammation-induced prostate cancer development. Results from recent studies have supported the theory that $C$. acnes may influence prostate cancer development by causing a chronic inflammation of the prostate [16, 18-21]. In line with the suggested role of $C$. acnes in prostate cancer development, our group reported the bacterium to be significantly more common in prostate tissue obtained from men with prostate cancer compared to men without the disease [16].

Additional findings supporting the hypothesis of $C$. acnes as a trigger of prostate cancer development comes from in vitro studies reporting an increased secretion of IL6 and CXCL8 by prostate epithelial cells infected with $C$. acnes $[16,21,22]$. Alexeyev et al. further detected $C$. acnes in prostate samples from individual patients taken up to six years apart, indicating a chronic $C$. acnes infection [23]. This is possibly facilitated by a capacity of $C$. acnes to evade eradication by immune cells [24-26]. A low-grade chronic inflammation caused by prostatic $C$. acnes infection, including induced secretion of IL6 and CXCL8, may thus over time contribute to prostate cancer development.

In order to evaluate whether prostatic $C$. acnes infection activates a systemic inflammatory response, or rather contributes to a low-grade localized inflammation of the prostate, the aim of the present study was to investigate whether prostatic $C$. acnes infections influence serum levels of IL6 and CXCL8 in prostate cancer patients.

\section{Material and methods}

\section{Study population}

A total number of 99 consecutive patients undergoing radical prostatectomy at Örebro University Hospital between 2009 and 2012 were included in this study. The techniques for biopsy and C. acnes characterization have been described in detail previously [16]. Prostate biopsies were obtained under sterile conditions at the operating theatre, after extraction of prostatectomy specimens. Culture and species verification of $C$. acnes were performed using routine microbiological procedures. $C$. acnes was isolated in samples from $61 \%$ of the patients $(n=60)$. Baseline characteristics were obtained from patient files and pathology reports. The study was approved by the regional ethical review board, Uppsala, Sweden (reference 2008/293).

\section{ELISA-analysis}

In order to investigate the serum levels of IL6 and CXCL8 in men with and without prostatic C. acnes infection, we collected $10 \mathrm{ml}$ blood from each patient prior to surgery. Blood samples were allowed to clot for $30 \mathrm{~min}$ at room temperature (RT) and serum was obtained by centrifugation at $3000 x \mathrm{~g}$ for $10 \mathrm{~min}$ at RT. Serum samples were then aliquoted and stored at -80 $\mathrm{C}$ pending analysis. All samples were subsequently analyzed retrospectively, and the individual performing measurements was blinded to $C$. acnes infection status of the respective study participants.

Serum samples were thawed on ice, and concentrations of IL6 and CXCL8 were measured using commercially available ELISA kits (ELISA Quantikine HS, catalog numbers HS600B (IL6) and HS800 (CXCL8), R\&D systems, Minneapolis, USA), according to manufacturer's instructions. The minimum detectable levels were $0.11 \mathrm{pg} / \mathrm{ml}$ for IL6 and $0.4 \mathrm{pg} / \mathrm{ml}$ for CXCL8, and the detection ranges were $0.2-10 \mathrm{pg} / \mathrm{ml}$ and $1-64 \mathrm{pg} / \mathrm{ml}$ respectively. The optical density was measured using a Multiskan Ascent plate reader (Thermo labsystems, Helsinki, Finland) at $490 \mathrm{~nm}$ and $620 \mathrm{~nm}$. The reference absorbance at $620 \mathrm{~nm}$ was subtracted from $450 \mathrm{~nm}$ in order to correct for background noise.

All samples were measured in duplicates, and samples where re-measured if one of the replicates failed to be measured. Blanks and standards were assayed according to the manufacturer's instructions. The mean values of absorbance vs. concentrations were plotted and a 4-parameter logistic (4PL) non-linear regression model fit was applied, and $\mathrm{R}^{2}$-values above 0.9 were considered as acceptable.

\section{Statistical analysis}

Mean replicate serum concentrations of IL6 and CXCL8 in each sample were used in the statistical analyses. Descriptive statistics included frequencies, proportions, medians and ranges. We used QQ-plots, histograms, box plots and Shapiro-Wilk Tests to evaluate continuous variables for normality. Prostate specific antigen (PSA), age at surgery, prostate volume (ultrasound-estimated), IL6, and CXCL8 were all found to be non-normally distributed. Differences between groups were analyzed using Chi-square Test, Mann-Whitney U Test or Kruskal-Wallis Test, where applicable. Associations between continuous variables were evaluated using non-parametric correlation, as well as by categorizing the exposure variable into quartiles and comparing medians of outcome variable. 
To test for confounding by age or other factors of the association between prostatic $C$. acnes infection and serum levels of CXCL8 and IL6 we performed simple and multiple linear regression analyses with CXCL8 and IL6 as continuous outcome variables. Outcome variables were transformed using the natural logarithm in order to normalize distribution. Outlying observations of $>3$ inter-quartile ranges (IQRs) were further excluded $(n=4$ for CXCL8, $n=0$ for IL6). Assumptions of normality, homoscedasticity and linear relationship between exposure and outcome were assessed visually using histogram of residual distributions, P-P plots, and plots of residuals vs. predicted values and exposure variables respectively. Influence diagnostics were performed using Cook's distance and multicollinearity was assessed using variance inflation factors (VIF). The simple linear regression models included $C$. acnes status as exposure and CXCL8/IL6 respectively as outcome. The multiple linear regression included Gleason sum (categorical; 6, 3+4, 4 $+3,8+$ ), age (continuous, years), PSA (continuous, ng/ $\mathrm{ml}$, transformed using the natural logarithm) and prostate volume ( $\mathrm{ml}$, continuous). We also tried a multiple linear regression model with continuous variables transformed into categorical variables, by dividing into quartiles. Covariates included in model were selected based on biological plausibility. All analyses were performed using IBM SPSS Statistics version 22 (IBM Corp. Armonk, NY, USA), and statistical significance was defined as $p<0.05$.

Based on previous observations of serum levels and standard deviations (SD) of IL6 and CXCL8 in healthy individuals $[27,28]$, the population size of the current study, and assuming a $\beta$ of 0.2 , the study is powered to detect an effect size of $0.39 \mathrm{SD}$. This corresponds to at least a medium effect size ( $>0.5 \mathrm{SD}$ ) as defined by Cohen [29].

\section{Results}

Baseline characteristics

A total number of 99 patients were eligible for analyses. The median age of the cohort was 64.6 years (range: 53.8-71.6 years), and the median preoperative PSA was $6.3 \mathrm{ng} / \mathrm{ml}$ (range: $2.1-43.0 \mathrm{ng} / \mathrm{ml}$ ). The Gleason score (GS) distribution of the patients was: GS $6: n=36$, GS 7: $n=59$ (GS $3+4: n=43$, GS $4+3: n=16)$, GS $8: n=2$, GS 9: $\mathrm{n}=2$, and GS 10: $n=0$. The pathologic stages were pT2 $(n=88)$ and pT3 $(n=11)$, and 34 patients had positive surgical margins. Men with prostatic $C$. acnes infection were on average slightly older than those without $C$. acnes infection (median age 65.1 vs. 63.1 years, $p=$ 0.047). Gleason score, PSA, pT-stage, ultra-sound estimation of prostate volume, and frequency of positive surgical margins did not differ between patients positive or negative for prostatic C. acnes infection (Table 1).
Table 1 Chararacteristics of study participants $(n=99)$, categorized by negative or positive finding of $C$. acnes

\begin{tabular}{|c|c|c|c|}
\hline & $\begin{array}{l}\text { C. acnes }+ \\
(n=60)\end{array}$ & $\begin{array}{l}\text { C. acnes- } \\
(n=39)\end{array}$ & $p$-value \\
\hline \multicolumn{4}{|c|}{ Age at time of surgery, years } \\
\hline Median (min-max) & $65.1(54.0-71.0)$ & $63.1(53.8-71.6)$ & $p=0.047^{a}$ \\
\hline \multicolumn{4}{|c|}{ PSA before surgery, $\mathrm{ng} / \mathrm{ml}$} \\
\hline Median (min-max) & $6.4(2.1-43)$ & $5.9(3.2-28)$ & $p=0.450^{\mathrm{a}}$ \\
\hline \multicolumn{4}{|l|}{ Prostate volume, $\mathrm{ml}$} \\
\hline Median (min-max) & $32(10-138)$ & $35(17-115)$ & $p=0.314^{a}$ \\
\hline \multicolumn{4}{|c|}{ Gleason score from prostatectomy sample, n (\%) } \\
\hline Gleason 6 & $25(41.7 \%)$ & $11(28.2 \%)$ & $p=0.170^{b}$ \\
\hline Gleason $3+4=7$ & $25(41.7 \%)$ & $18(46.2 \%)$ & \\
\hline Gleason $4+3=7$ & $8(13.3 \%)$ & $8(20.5 \%)$ & \\
\hline Gleason 8 & $2(3.3 \%)$ & $0(0 \%)$ & \\
\hline Gleason 9-10 & $0(0 \%)$ & $2(5.1 \%)$ & \\
\hline $\begin{array}{l}\text { Positive surgical } \\
\text { margins n (\%) }\end{array}$ & $22(36.7 \%)$ & $12(30.8 \%)$ & $p=0.546^{b}$ \\
\hline \multicolumn{4}{|l|}{ pT-stage, n (\%) } \\
\hline pT2a & $8(13.3 \%)$ & $5(12.8 \%)$ & $p=0.149^{b}$ \\
\hline pT2c & $46(76.7 \%)$ & $29(74.4 \%)$ & \\
\hline pT3a & $6(10.0 \%)$ & $2(5.1 \%)$ & \\
\hline pT3b & $0(0 \%)$ & $3(7.7 \%)$ & \\
\hline
\end{tabular}

${ }^{a}$ Mann-Whitney $\mathrm{U}$ test

${ }^{\mathrm{b}}$ Chi-square test

\section{Serum levels of IL6 and CXCL8 in relation to various patient characteristics}

Due to insufficient amount of serum, we were unable to measure concentrations of IL6 in one sample and CXCL8 in four additional samples. For patients with available cytokine data, the median pre-operative serum levels of IL6 and CXCL8 were $1.09 \mathrm{pg} / \mathrm{ml}$ (range 0.30$3.76 \mathrm{pg} / \mathrm{ml}$ ) and $9.97 \mathrm{pg} / \mathrm{ml}$ (range $3.36-155.53 \mathrm{pg} / \mathrm{ml}$ ), respectively. Median serum levels of IL6 and CXCL8 did not differ when comparing groups based on GS, pT-stage, surgical margins, PSA, age and prostate volume (Table 2 and Table 3). Non-parametric correlation did further not indicate any significant correlation between IL6 or CXCL8 and age, PSA or prostate volume respectively (Table 2 and Table 3 ).

\section{Serum levels of IL6 and CXCL8 in men with and without prostatic $C$. acnes infection}

Median levels of IL6 for patients with and without prostatic $C$. acnes infection were $1.04 \mathrm{pg} / \mathrm{ml}$ and $1.16 \mathrm{pg} / \mathrm{ml}$, respectively (Table 2). For CXCL8, the corresponding values were $10.01 \mathrm{pg} / \mathrm{ml}$ and $9.68 \mathrm{pg} / \mathrm{ml}$ (Table 3). Comparison of patients with and without prostatic $C$. acnes infection did not indicate statistically significant differences in serum levels of IL6 or CXCL8. 
Table 2 Preoperative serum levels of IL6 $(\mathrm{pg} / \mathrm{ml})$ in relation to other variables for men who underwent radical prostatectomy and had available information on serum IL6 $(n=98)$

\begin{tabular}{|c|c|c|c|c|c|}
\hline \multicolumn{6}{|l|}{ Total population $(n=98)$} \\
\hline Median serum IL6, pg/ml (min-max) & $1.09(0.30-3.76)$ & & & & \\
\hline By C. acnes category & C. acnes $+(n=60)$ & & C. acnes- $(n=38)$ & & \\
\hline Median serum IL6, pg/ml (min-max) & $1.04(0.35-3.76)$ & & $1.16(0.30-3.13)$ & & $p=0.106^{a}$ \\
\hline Gleason sum & Gleason $6(n=36)$ & Gleason $3+4(n=42)$ & Gleason $4+3(n=16)$ & Gleason $8+(n=4)$ & \\
\hline Median serum IL6, pg/ml (min-max) & $1.02(0.3-3.76)$ & $1.09(0.45-3.13)$ & $1.14(0.47-2.05)$ & $1.12(0.7-1.37)$ & $p=0.836^{a}$ \\
\hline Positive sugical margins & No $(n=64)$ & & Yes $(n=34)$ & & \\
\hline Median serum IL6, pg/ml (min-max) & $1.12(0.3-3.76)$ & & $1.03(0.39-2.97)$ & & $p=0.420^{\mathrm{a}}$ \\
\hline pT stage & pT2 $(n=87)$ & & pT3 $(n=11)$ & & \\
\hline Median serum IL6, pg/ml (min-max) & $1.1(0.3-3.76)$ & & $1.05(0.61-2.06)$ & & $p=0.862^{a}$ \\
\hline \multicolumn{6}{|l|}{ PSA } \\
\hline By PSA quartile & $<4.6 \mathrm{ng} / \mathrm{ml}$ & $\geq 4.6,<6.4 \mathrm{ng} / \mathrm{ml}$ & $\geq 6.4,<9.0 \mathrm{ng} / \mathrm{ml}$ & $\geq 9 \mathrm{ng} / \mathrm{ml}$ & \\
\hline Median serum IL6, pg/ml (min-max) & $1.02(0.45-2.88)$ & $1.08(0.47-2.23)$ & $1.12(0.3-3.13)$ & $1.16(0.39-3.76)$ & $p=0.601^{a}$ \\
\hline \multicolumn{6}{|l|}{ Non-parametric correlation } \\
\hline Correlation coefficient & 0.058 & & & & $p=0.571^{b}$ \\
\hline \multicolumn{6}{|l|}{ Age } \\
\hline By age quartile & $<61.3$ years & $\geq 61.3,<64.7$ years & $\geq 64.7,<67.1$ years & $\geq 67.1$ years & \\
\hline Median serum IL6, pg/ml (min-max) & $1.09(0.3-3.13)$ & $0.93(0.39-2.97)$ & $1.17(0.47-3.76)$ & $1.11(0.35-2.88)$ & $p=0.416^{\mathrm{a}}$ \\
\hline \multicolumn{6}{|l|}{ Non-parametric correlation } \\
\hline Correlation coefficient & 0.113 & & & & $p=0.270^{b}$ \\
\hline \multicolumn{6}{|l|}{ Prostate volume } \\
\hline By volume quartile & $10-26 \mathrm{ml}$ & $27-33 \mathrm{ml}$ & $34-50 \mathrm{ml}$ & $51-138 \mathrm{ml}$ & \\
\hline Median serum IL6, pg/ml (min-max) & $1.39(0.3-3.76)$ & $1.1(0.56-2.68)$ & $1.06(0.39-2.73)$ & $1.1(0.35-2.78)$ & $p=0.397^{\mathrm{a}}$ \\
\hline \multicolumn{6}{|l|}{ Non-parametric correlation } \\
\hline Correlation coefficient & -0.106 & & & & $p=0.321^{b}$ \\
\hline
\end{tabular}

Simple linear regression did not indicate significant association between $C$. acnes status and IL6 (log-transformed); regerssion coefficient $(\beta)$ : $-0.17 ; 95 \%$ Confidence interval (95\% CI): $-0.39,0.06 ; p=0.143$. Adjustment for multiple covariates did not change coefficients markedly $(\beta$ : $-0.17,95 \% \mathrm{CI}:-0.43,0.09, p=$ 0.187). The regression coefficient for simple linear regression of $C$. acnes on CXCL8 (log-transformed) was $0.11(95 \% \mathrm{CI}-0.07,0.30, p=0,234)$ and for the multivariable model 0.09 (95\% CI -0.11, 029, $p=$ 0,361 , likewise suggesting absence of association. Exclusion of individual covariates, and categorization of model covariates did not bring about significant changes in parameter estimates (data not shown). Regression diagnostics did not indicate violation of assumptions after transformation of outcome variables and exclusion of outliers in CXCL8. In summary, we did not find evidence for confounding of the association between $C$. acnes and serum levels of CXCL8 and IL6, and no further evidence of a significant association.

\section{Discussion}

Previous studies have shown that $C$. acnes has the capability to stimulate secretion of IL6 and CXCL8 by prostate epithelial cells in vitro [16, 21, 22], and a microenvironment containing high levels of these two inflammatory mediators has been associated with prostate cancer progression $[6-8,10,11]$. In the present study, we aimed to evaluate the influence of prostatic $C$. acnes infection on systemic levels of IL6 and CXCL8 in prostate cancer patients. When comparing patients with and without prostatic $C$. acnes infection, no significant differences were observed in serum levels of either mediator.

Chronic inflammation is considered involved in the development of several types of cancer [1], and multiple lines of evidence indicate that inflammation may influence prostate cancer development and progression [2]. Histological signs of prostatic inflammation are frequently observed [3, 4], and the term proliferative inflammatory atrophy (PIA) has been coined for areas characterized by atrophy, inflammation and 
Table 3 Preoperative serum levels of $\mathrm{CXCL8}(\mathrm{pg} / \mathrm{ml})$ in relation to other variables for men who underwent radical prostatectomy and had available information on serum CXCL8 $(n=95)$

\begin{tabular}{|c|c|c|c|c|c|}
\hline \multicolumn{6}{|l|}{ Total population $(n=95)$} \\
\hline Median serum CXCL8, pg/ml (min-max) & $9.97(3.36-155.53)$ & & & & \\
\hline By C. acnes category & C. acnes $+(n=58)$ & & C. acnes- $(n=37)$ & & \\
\hline Median serum $\mathrm{CXCL8}$, pg/ml (min-max) & $10.01(5.05-130.93)$ & & $9.68(3.36-155.53)$ & & $p=0.658^{\mathrm{a}}$ \\
\hline Gleason sum & Gleason $6(n=36)$ & Gleason $3+4(n=41)$ & Gleason $4+3(n=15)$ & Gleason $8+(n=3)$ & \\
\hline Median serum CXCL8, pg/ml (min-max) & $10.05(4-155.53)$ & $9.21(3.36-83.2)$ & $9.96(5.95-16.07)$ & $11.16(9.41-12.14)$ & $p=0.843^{a}$ \\
\hline Positive sugical margins & No $(n=62)$ & & Yes $(n=33)$ & & \\
\hline Median serum CXCL8, pg/ml (min-max) & $10.01(3.36-155.53)$ & & $9.41(4.79-38.16)$ & & $p=0.344^{\mathrm{a}}$ \\
\hline pT stage & pT2 $(n=86)$ & & pT3 $(n=9)$ & & \\
\hline Median serum $\mathrm{CXCL8}$, pg/ml (min-max) & $9.99(3.36-155.53)$ & & $9.41(6.24-20.53)$ & & $p=0.576^{\mathrm{a}}$ \\
\hline \multicolumn{6}{|l|}{ PSA } \\
\hline By PSA quartile & $<4.6 \mathrm{ng} / \mathrm{ml}$ & $\geq 4.6,<6.4 \mathrm{ng} / \mathrm{ml}$ & $\geq 6.4,<9.0 \mathrm{ng} / \mathrm{ml}$ & $\geq 9 \mathrm{ng} / \mathrm{ml}$ & \\
\hline Median serum CXCL8, pg/ml (min-max) & $9,68(5,05-130,93)$ & $10,03(4,79-155,53)$ & $8,93(4-38,16)$ & $10,65(3,36-83,2)$ & $p=0.646^{\mathrm{a}}$ \\
\hline \multicolumn{6}{|l|}{ Non-parametric correlation } \\
\hline Correlation coefficient & 0.069 & & & & $p=0.505^{b}$ \\
\hline \multicolumn{6}{|l|}{ Age } \\
\hline By age quartile & $<61.3$ years & $\geq 61.3,<64.7$ years & $\geq 64.7,<67.1$ years & $\geq 67.1$ years & \\
\hline Median serum CXCL8, pg/ml (min-max) & $10,09(3,36-155,53)$ & $10,09(3,36-155,53)$ & $9,21(5,26-83,2)$ & $11,16(5,4-62,26)$ & \\
\hline \multicolumn{6}{|l|}{ Non-parametric correlation } \\
\hline Correlation coefficient & 0.044 & & & & $p=0.674^{b}$ \\
\hline \multicolumn{6}{|l|}{ Prostate volume } \\
\hline By volume quartile & $0-26 \mathrm{ml}$ & $27-33 \mathrm{ml}$ & $34-50 \mathrm{ml}$ & $51-138 \mathrm{ml}$ & \\
\hline Median serum CXCL8, pg/ml (min-max) & $10.28(6.24-16.68)$ & $8.89(3.36-83.2)$ & $10.8(4-130.93)$ & $9.68(5.26-155.53)$ & $p=0.468^{a}$ \\
\hline \multicolumn{6}{|l|}{ Non-parametric correlation } \\
\hline Correlation coefficient & 0.045 & & & & $p=0.681^{b}$ \\
\hline
\end{tabular}

${ }^{a} p$-value fron Kruskal-Wallis Test/Mann-Whitney U Test; ${ }^{b} p$-value from Spearman correllation

epithelial proliferation [30]. Morphologic studies have indicated transition from PIA to high-grade prostatic intraepithelial neoplasia (HGPIN, an established precursor lesion to prostate cancer), and possibly also to prostate cancer [31,32]. Epidemiological studies and genetic association studies have presented further evidence supporting a possible association between prostatic inflammation and prostate cancer development $[9,33]$.

Based on its prevalence in prostate specimens $[14-16,21]$, and on observed associations with prostatic inflammation [17, 22, 34, 35], C. acnes has been proposed as an etiological agent for prostatic inflammation. Observed associations between prostatic occurrence of $C$. acnes and prostate cancer have consequently led to the hypothesis that prostatic inflammation caused by $C$. acnes might influence prostate carcinogenesis $[16,18,21]$. There is further some epidemiological support for a positive association between acne vulgaris and prostate cancer risk $[19,20]$.
IL6 and CXCL8 are pro-inflammatory mediators with diverse roles in the innate immune system. IL6 is a pleiotropic molecule involved in regulation of acute phase inflammatory response and T-cell differentiation [36], and CXCL8 is associated with chemotaxis and activation of granulocytes [37]. In addition to their role in innate immune system functions, such as protection against bacterial infections, cytokines and chemokines have been proposed to contribute to cancer development and progression. Specifically, IL6 and CXCL8 have been extensively studied in relation to prostate cancer, and have both been proposed to play important roles in prostate cancer development and progression $[6-8,10,11]$. C. acnes has in several studies, including one using prostate-derived isolates from the present cohort, been observed to induce secretion of both IL6 and CXCL8 by prostate epithelial cells in vitro $[16,21,22]$.

The fact that we did not detect a significant difference in serum levels of IL6 or CXCL8 in patients with and 
without prostatic $C$. acnes infection indicates that $C$. acnes is not capable of inducing a systemic response of IL6 and CXCL8. These results are in line with previous studies evaluating serum levels of IL6 as a diagnostic marker for C. acnes-related peri-prosthetic shoulder infections. The indolent nature of $C$. acnes infection has been proposed as a possible reason for the lack of systemic effects $[38,39]$. Studies on acne vulgaris likewise suggest absence of systemic inflammatory response, estimated by $\mathrm{C}$-reactive protein (CRP) level, even in severe cases [40]. It should however be noted that elevated levels of CRP was observed previously in an animal model for prostatic C. acnes infection [34].

The lack of a systemic response further conforms to evidence indicating a capacity of $C$. acnes to avoid detection and eradication by the immune system, and thereby cause a persistent infection. Resistance to killing by neutrophils and macrophages [26], evasion of phagosomal degradation and long-time intracellular survival in macrophages [23-25, 41], as well as biofilm formation $[13,23]$ constitute possible mechanisms by which the bacterium avoids elimination. Further, Sahdo et al. reported low Caspase-1 activity in macrophages when $C$. acnes isolates, cultivated from patients in the present cohort, were used as stimuli for peripheral leukocytes obtained from blood donors [42]. The same study also observed that commensal $C$. acnes isolates induced Caspase- 1 activity to a greater degree than invasive strains.

A strength with this study is the demonstrated capacity of $C$. acnes, cultured from men included in the present cohort, to induce IL6 and CXCL8 secretion by prostate epithelial cells [16]. Additional strengths of the study include serum samples obtained contemporaneously with prostate samples for microbiological analysis, as well as a relatively large study population compared to other studies of inflammatory mediators in relation to C. acnes pathology $[38,39]$. The study, however, still lacks power to detect small differences in serum levels of IL6 and CXCL8. Study limitations include the lack of CRP as a measure of systemic inflammation, and the use of only two inflammatory mediators. Future studies may consider including multiple pro- as well as anti-inflammatory mediators in order to better characterize the systemic inflammatory response to a $C$. acnes infection.

As previously reported, prostatic $C$. acnes infection was in this cohort associated with prostate cancer in a case-control setting, but not with GS, tumor stage, or PSA [16]. In the present study, we did not observe a difference in serum levels of IL6 or CXCL8 between patients with or without prostatic $C$. acnes infection. The results are consistent with $C$. acnes proposed capacity to colonize the prostate and induce a low-grade chronic prostatic inflammation, with little effect on systemic inflammatory response, but with possible implications for prostate cancer development. Evading inflammatory activation may facilitate $C$. acnes' persistence and long-term survival in prostatic tissue.

\section{Conclusions}

Our results indicate that $C$. acnes infection of the prostate does not give rise to a measurable increase in systemic levels of IL6 and CXCL8. This is consistent with previous observations of $C$. acnes giving rise to chronic low-grade infections.

\section{Abbreviations \\ 4PL: 4-parameter logistic; C. acnes: Cutibacterium acnes; CRP: C-reactive protein; CXCL8: C-X-C motif chemokine ligand 8; GS: Gleason score; HGPIN: High-grade prostatic intraepithelial neoplasia; IL6: Interleukin 6; PIA: Proliferative inflammatory atrophy; PSA: Prostate specific antigen; RT: Room temperature; SD: Standard deviation}

\section{Acknowledgements \\ Not applicable. \\ Funding \\ The project was funded by the foundation Lions Cancerforkkningsfond vid Akademiska sjukhuset i Uppsla.}

\section{Availability of data and materials}

The dataset generated and analyzed during the current study is not publicly available, due to confidentiality issues regarding patient-related information, but is available from the corresponding author on reasonable request.

\section{Authors' contributions}

OA participated in initial project conception, supervision and collection of prostate tissue. JC and SD participated in project conception and collected tissue for microbiological analysis. They further supervised laboratory work, data interpretation and manuscript preparation. KF provided conceptual and analytic support for the project, and assisted manuscript preparation. HU performed ELISA-analyses and statistical analysis, conducted data interpretation and prepared manuscript. BS participated in initial conception of the project, and supervised microbiological analysis. All authors read and approved the final manuscript.

\section{Ethics approval and consent to participate}

The study was approved by the regional ethical review board, Uppsala, Sweden (reference 2008/293). All study participants provided written and oral consent to study participation.

Consent for publication

Not applicable.

\section{Competing interests}

The authors declare that they have no competing interests.

\section{Publisher's Note}

Springer Nature remains neutral with regard to jurisdictional claims in published maps and institutional affiliations.

\section{Author details}

'Department of Urology, Faculty of Medicine and Health, Örebro University, 70185 Örebro, Sweden. ${ }^{2}$ Faculty of Medicine and Health, Örebro University, Örebro, Sweden. ${ }^{3}$ Clinical Epidemiology and Biostatistics, School of Medical Sciences, Örebro University, Örebro, Sweden. ${ }^{4}$ Department of Medical Epidemiology, Karolinska Institutet, Stockholm, Sweden. 
Received: 26 June 2018 Accepted: 12 October 2018

Published online: 14 November 2018

\section{References}

1. Coussens LM, Werb Z. Inflammation and cancer. Nature. 2002;420:860-7.

2. Sfanos KS, De Marzo AM. Prostate cancer and inflammation: the evidence. Histopathology. 2012;60:199-215.

3. Di Silverio F, Gentile V, De Matteis A, Mariotti G, Giuseppe V, Luigi PA, Sciarra A. Distribution of inflammation, pre-malignant lesions, incidental carcinoma in histologically confirmed benign prostatic hyperplasia: a retrospective analysis. Eur Urol. 2003:43:164-75.

4. Gerstenbluth RE, Seftel AD, MacLennan GT, Rao RN, Corty EW, Ferguson K, Resnick MI. Distribution of chronic prostatitis in radical prostatectomy specimens with up-regulation of bcl-2 in areas of inflammation. J Urol. 2002;167:2267-70.

5. Schatteman PH, Hoekx L, Wyndaele JJ, Jeuris W, Van Marck E. Inflammation in prostate biopsies of men without prostatic malignancy or clinical prostatitis: correlation with total serum PSA and PSA density. Eur Urol. 2000; 37:404-12.

6. Puhr M, De Marzo A, Isaacs W, Lucia MS, Sfanos K, Yegnasubramanian S, Culig Z. Inflammation, microbiota, and prostate Cancer. Eur Urol Focus. 2016;2:374-82

7. Araki S, Omori Y, Lyn D, Singh RK, Meinbach DM, Sandman Y, Lokeshwar VB, Lokeshwar BL. Interleukin-8 is a molecular determinant of androgen independence and progression in prostate cancer. Cancer Res. 2007;67: 6854-62.

8. Moore BB, Arenberg DA, Stoy K, Morgan T, Addison CL, Morris SB, Glass M, Wilke C, Xue YY, Sitterding S, et al. Distinct CXC chemokines mediate tumorigenicity of prostate cancer cells. Am J Pathol. 1999;154:1503-12.

9. Tindall EA, Hayes VM, Petersen DC. Inflammatory genetic markers of prostate cancer risk. Cancers (Basel). 2010;2:1198-220.

10. Nguyen DP, Li J, Tewari AK. Inflammation and prostate cancer: the role of interleukin 6 (IL-6). BJU Int. 2014;113:986-92.

11. Shariat SF, Andrews B, Kattan MW, Kim J, Wheeler TM, Slawin KM. Plasma levels of interleukin-6 and its soluble receptor are associated with prostate cancer progression and metastasis. Urology. 2001;58:1008-15.

12. Das S, Reynolds RV. Recent advances in acne pathogenesis: implications for therapy. Am J Clin Dermatol. 2014:15:479-88.

13. Achermann Y, Goldstein EJ, Coenye T, Shirtliff ME. Propionibacterium acnes: from commensal to opportunistic biofilm-associated implant pathogen. Clin Microbiol Rev. 2014;27:419-40.

14. Alexeyev O, Bergh J, Marklund I, Thellenberg-Karlsson C, Wiklund F, Gronberg $H$, Bergh A, Elgh F. Association between the presence of bacterial $16 \mathrm{~S}$ RNA in prostate specimens taken during transurethral resection of prostate and subsequent risk of prostate cancer (Sweden). Cancer Causes Control. 2006;17:1127-33.

15. Cavarretta I, Ferrarese R, Cazzaniga W, Saita D, Luciano R, Ceresola ER, Locatelli I, Visconti L, Lavorgna G, Briganti A, et al. The microbiome of the prostate tumor microenvironment. Eur Urol. 2017.

16. Davidsson S, Molling P, Rider JR, Unemo M, Karlsson MG, Carlsson J, Andersson SO, Elgh F, Soderquis B, Andren O. Frequency and typing of Propionibacterium acnes in prostate tissue obtained from men with and without prostate cancer. Infect Agent Cancer. 2016;11:26.

17. Cohen RJ, Shannon BA, McNeal JE, Shannon T, Garrett KL. Propionibacterium acnes associated with inflammation in radical prostatectomy specimens: a possible link to cancer evolution? J Urol. 2005:173:1969-74.

18. Kakegawa T, Bae Y, Ito T, Uchida K, Sekine M, Nakajima Y, et al. Frequency of Propionibacterium acnes Infection in Prostate Glands with Negative Biopsy Results Is an Independent Risk Factor for Prostate Cancer in Patients with Increased Serum PSA Titers. PloS one. 2017:12(1):e0169984.

19. Sutcliffe $S$, Giovannucci E, Isaacs WB, Willett WC, Platz EA. Acne and risk of prostate cancer. Int J Cancer. 2007;121:2688-92.

20. Ugge H, Udumyan R, Carlsson J, Andren O, Montgomery S, Davidsson S, et al. Acne in late adolescence and risk of prostate cancer. Int J Cancer. 2018; 142(8):1580-5

21. Fassi Fehri L, Mak TN, Laube B, Brinkmann V, Ogilvie LA, Mollenkopf H, Lein M, Schmidt T, Meyer TF, Bruggemann H. Prevalence of Propionibacterium acnes in diseased prostates and its inflammatory and transforming activity on prostate epithelial cells. Int J Med Microbiol. 2011;301:69-78.

22. Drott JB, Alexeyev O, Bergstrom P, Elgh F, Olsson J. Propionibacterium acnes infection induces upregulation of inflammatory genes and cytokine secretion in prostate epithelial cells. BMC Microbiol. 2010;10:126.
23. Alexeyev OA, Marklund I, Shannon B, Golovleva I, Olsson J, Andersson C, Eriksson I, Cohen R, Elgh F. Direct visualization of Propionibacterium acnes in prostate tissue by multicolor fluorescent in situ hybridization assay. J Clin Microbiol. 2007:45:3721-8.

24. Bae $Y$, Ito T, lida T, Uchida K, Sekine M, Nakajima Y, Kumagai J, Yokoyama T, Kawachi H, Akashi T, Eishi Y. Intracellular Propionibacterium acnes infection in glandular epithelium and stromal macrophages of the prostate with or without cancer. PLoS One. 2014:9:e90324.

25. Fischer N, Mak TN, Shinohara DB, Sfanos KS, Meyer TF, Bruggemann H. Deciphering the intracellular fate of Propionibacterium acnes in macrophages. Biomed Res Int. 2013;2013:603046.

26. Webster GF, Leyden JJ, Musson RA, Douglas SD. Susceptibility of Propionibacterium acnes to killing and degradation by human neutrophils and monocytes in vitro. Infect Immun. 1985;49:116-21.

27. Cava F, Gonzalez C, Pascual MJ, Navajo JA, Gonzalez-Buitrago JM. Biological variation of interleukin 6 (IL-6) and soluble interleukin 2 receptor (sIL2R) in serum of healthy individuals. Cytokine. 2000;12:1423-5.

28. Gonzalez C, Cava F, Ayllon A, Guevara P, Navajo JA, Gonzalez-Buitrago JM. Biological variation of interleukin-1 beta, interleukin-8 and tumor necrosis factoralpha in serum of healthy individuals. Clin Chem Lab Med. 2001:39:836-41.

29. Sullivan GM, Feinn R. Using effect size-or why the $P$ value is not enough. $J$ Grad Med Educ. 2012:4:279-82.

30. De Marzo AM, Marchi VL, Epstein JI, Nelson WG. Proliferative inflammatory atrophy of the prostate: implications for prostatic carcinogenesis. Am J Pathol. 1999;155:1985-92.

31. De Marzo AM, Nakai Y, Nelson WG. Inflammation, atrophy, and prostate carcinogenesis. Urol Oncol. 2007;25:398-400.

32. Wang W, Bergh A, Damber JE. Morphological transition of proliferative inflammatory atrophy to high-grade intraepithelial neoplasia and cancer in human prostate. Prostate. 2009;69:1378-86.

33. Sutcliffe S, Platz EA. Inflammation in the etiology of prostate cancer: an epidemiologic perspective. Urol Oncol. 2007;25:242-9.

34. Olsson J, Drott JB, Laurantzon L, Laurantzon O, Bergh A, Elgh F. Chronic prostatic infection and inflammation by Propionibacterium acnes in a rat prostate infection model. PLoS One. 2012;7:e51434.

35. Shinohara DB, Vaghasia AM, Yu SH, Mak TN, Bruggemann H, Nelson WG, De Marzo AM, Yegnasubramanian S, Sfanos KS. A mouse model of chronic prostatic inflammation using a human prostate cancer-derived isolate of Propionibacterium acnes. Prostate. 2013;73:1007-15.

36. Neurath MF, Finotto S. IL-6 signaling in autoimmunity, chronic inflammation and inflammation-associated cancer. Cytokine Growth Factor Rev. 2011;22:83-9.

37. Waugh DJ, Wilson C. The interleukin-8 pathway in cancer. Clin Cancer Res. 2008;14:6735-41.

38. Frangiamore SJ, Saleh A, Kovac MF, Grosso MJ, Zhang X, Bauer TW, Daly TM, Ricchetti ET, lannotti JP. Synovial fluid interleukin-6 as a predictor of periprosthetic shoulder infection. J Bone Joint Surg Am. 2015;97:63-70.

39. Grosso MJ, Frangiamore SJ, Saleh A, Kovac MF, Hayashi R, Ricchetti ET, Bauer TW, lannotti JP. Poor utility of serum interleukin-6 levels to predict indolent periprosthetic shoulder infections. J Shoulder Elb Surg. 2014;23:1277-81.

40. Namazi MR, Parhizkar AR, Jowkar F. Serum levels of hypersensitive-C-reactive protein in moderate and severe acne. Indian Dermatol Online J. 2015;6:253-7.

41. Csukas Z, Banizs B, Rozgonyi F. Studies on the cytotoxic effects of Propionibacterium acnes strains isolated from cornea. Microb Pathog. 2004:36:171-4.

42. Sahdo B, Sarndahl E, Elgh F, Soderquist B. Propionibacterium acnes activates caspase-1 in human neutrophils. Apmis. 2013;121:652-63.

\section{Ready to submit your research? Choose BMC and benefit from:}

- fast, convenient online submission

- thorough peer review by experienced researchers in your field

- rapid publication on acceptance

- support for research data, including large and complex data types

- gold Open Access which fosters wider collaboration and increased citations

- maximum visibility for your research: over $100 \mathrm{M}$ website views per year

At $\mathrm{BMC}$, research is always in progress.

Learn more biomedcentral.com/submissions 